\title{
Dünnschichtzytologie (liquid-based cytology) - Ende der Diskussion?
}

Siegfried Heinzl

Prof. Dr. med. Siegfried Heinzl Facharzt FMH für Geburtshilfe und Gynäkologie

speziell gynäkologische Onkologie Chefarzt Frauenklinik

Kantonsspital

CH-4101 Bruderholz

Tel. 0614362173

Fax 0614363666

siegfried.heinzl@ksbh.ch
Die Diskussion über die Vor- und Nachteile der Dünnschichtzytologie (liquid-based cytology LBC) hat auch in dieser Zeitschrift sehr unterschiedliche Reaktionen ausgelöst $[4,6,7,9,10]$. Man könnte fast behaupten, die betroffene Ärzteschaft wurde gespalten. Während die einen von den Vorzügen dieser Methode überzeugt sind und waren, beklagten die anderen, dass die Vorteile - wenn überhaupt - so marginal sind, dass sich die Mehrkosten nicht rechtfertigen. Mittlerweile liegen sehr viele Studien mit z.T. unterschiedlichen Resultaten vor [zit. in 1, 2, $7,8,9]$.

In der Schweiz wurde die Dünnschichtmethode vom BAG vorübergehend zugelassen [3]. Dies mit der Auflage, nachzuweisen, dass die Dünnschichtmethode der konventionellen Methode überlegen sei. Während dieser Zeit konnte für die Dünnschichtmethode im TARMED ein Zuschlag (37.0620) verrechnet werden. Nach eingehender Prüfung hat das BAG und die ELK in dieser leidigen Frage eine Entscheidung herbeigeführt. Dabei wurde folgendes ausgeführt: «Den Dünnschicht-Methoden (Thinprep und Autocyte Prep/SurePath) wurde von BAG und ELK keine Überlegenheit attestiert, sondern lediglich Gleichwertigkeit» [5].

Daraufhin wurde im TARMED auf Antrag von santésuisse der Dünnschichtzuschlag gestrichen, und seit dem 1. Juli 2005 besteht die Tarifgleichheit für beide Methoden. Somit gibt es keinen Grund mehr, die Dünnschichtmethoden nicht zu bezahlen. Sie sind seit dem 1. Juli 2005 als leistungspflichtig in Anhang 1 KLV aufgeführt, und zwar explizit auch für die Anwendung in der Screeningsituation [3]. Diese Lösung kann als gut-schweizerischer Kompromiss bezeichnet werden, können doch beide Seiten damit gut leben. Die Befürworter können «ihre Methode» weiterführen, während die Sorge der Gegner, dass die Kosten des Screenings weiter unnötig steigen, nicht mehr gegeben ist.

Mittlerweile wurde die Entscheidung des Bundesamtes für Gesundheit durch eine grossangelegte Metaanalyse bestätigt. Die im Januar 2006 im «Lancet» von E. Davey et al. publizierte Metaanalyse untersuchte 56 Primärstudien und kommt zu folgender Interpretation [2]: «We saw no evidence that liquid-based cytology reduced the proportion of unsatisfactoryslides, or detected more high-grade lesions in high-quality studies, than conventional cytology. This review does not lend support to claim of better performance by liquid-based cytology. Large randomised controlled trials are needed.»

In derselben Zeitschrift unterstreichen die zum Kommentar eingeladenen Herren Dres. med. J. Obwegeser, Zürich, und V. Schneider, Freiburg im Breisgau, dass die unterschiedliche Organisation wie auch die unterschiedliche Rechtslage die unterschiedlichen Ergebnisse erklären. Bei korrekter Abnahme des PAP-Abstrichs können sowohl mit der konventionellen wie auch mit der Dünnschichtmethode entsprechende Ergebnisse erzielt werden [8].

Für die Praxis bedeutet dies einerseits, dass zur TARMED-Position 37.0700 (Zytologie zur Krebsvorsorge, nicht-ärztliche Leistung: Objektträger von Portio-, Zervix-, Vagina-Abstrich zur zytologischen Krebsvorsorge) der Zuschlag 37.0620 (Zuschlag für Sedimentationspräparation oder Filtrationspräparation [Liquid-based-Methode]) nicht mehr verrechnet werden darf [11]. Andererseits darf die Methode angewandt werden, welche man für die richtige oder bessere erachtet. Dies soll aber nicht bedeuten, dass die Diskussion beendet ist. Sobald relevante neue Erkenntnisse vorliegen, muss die Diskussion wieder geführt werden.

In diesem Zusammenhang sei nochmals darauf hingewiesen, dass das viel grössere Problem bei der Bekämpfung des Zervixkarzinoms die Teilnahme am Zervixscreening darstellt als die hier diskutierte Abstrichentnahmemethodik und deren Verarbeitung.

Obwohl in der Schweiz entsprechende Zahlen fehlen, ist anzunehmen, dass viel mehr Zervixkarzinome durch die Nichtteilnahme am Screeningprogramm entstehen als durch Fehler der Abstrichentnahme- und -verarbeitungstechnik. 


\section{Literatur}

1 Arbeitsgruppe «Guideline Zervixabstrich». Guideline zum Vorgehen bei suspektem und positivem Abstrich der Cervix uteri. Überarbeitete Fassung, Version 2.4. Schweiz Ärztezeitung 2004; 85(43):2305-23.

2 Davey E, Barratt A, Irwig L, Chan SF, et al. Effect of study design and quality on unsatisfactory rates, cytology classifactions, and accuracy in liquidbased versus conventional cervical cytology: a systematic review. Lancet 2006;367:122-32.

3 Eidgenössisches Departement des Inneren (EDI). Verordnung über Leistungen in der obligatorischen Krankenpflegeversicherung. Stand 12. Juli 2005.

4 Feichter G. Warum es die Dünnschichtzytologie (nicht) braucht! Schweiz Ärztezeitung 2004; 85(49):2628-30.

5 Gurtner F. Persönliche Mitteilung. 21. März 2006.
6 Heinzl S. Vorgehen bei suspektem und positivem Abstrich der Cervix uteri. Replik zum Kommentar. Schweiz Ärztezeitung 2003;84(31):1631-2.

7 Obwegeser J. Warum es die Dünnschichtzytologie nicht braucht! Schweiz Ärztezeitung 2004; 85(10):512-14.

8 Obwegeser J, Schneider V. Thin-layer cervical cytology: a new meta-analysis. Lancet 2006; 367:88-9.

9 Spezialärztinnen und -ärzte für Zytopathologie. Dünnschichtzytologie. Schweiz Ärztezeitung 2003;84(50):2666-7.

10 Spycher B. Die Dünnschichtzytologie: zurück zum Wesentlichen. Schweiz Ärztezeitung 2004; 85(19):987-8

11 TARMED FAQ-Team. Persönliche Mitteilung. 7. November 2005 